\section{New \\ The foot inaudible and noiseless}

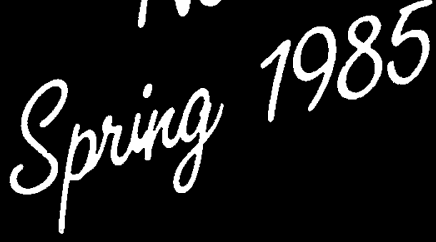

NUCLEOTIDE SEQUENCES 1985

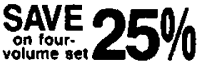

This vital reference work is the result of a unique collaboration between the EMBL Sequence Data Library staff, the staff of GenBank $^{\circledR}$ at Los Alamos National Laboratory, and Bolt Beranek and Newman Inc. It contains over $4,000,000$ bases from nearly 6,300 reported sequences. Keyword phrase, taxonomy, author and library location indexes assist the use of each volume.

Due April 1985. Set of four volumes: 2072pp; $094794625 \times$ (softbound); $£ 85.00 /$ US\$120.00.

I Primates and rodents: $524 p p$; 094794626 8; £28.00/US\$40.00

II Other eukaryotes: $474 p p$ :

0947946276 ; £28.00/US\$40.00.

III Bacteria, bacteriophage, organelles and structural RNA: 538pp;

$0947946284 ; \varepsilon 28.00 / U S \$ 40.00$

IV Viral and synthetic sequences:

536pp; $0947946292 ; £ 28.00 / U S \$ 40.00$

for those without computer access or for the occasional "Has this gene been sequenced?" enquiry, the printed compendium will be of great value. Nature (on Nucleotide Sequences 1984)

\section{Marihuana '84}

Proceedings of the Oxford Symposium on Cannabis

Edited by D J Harvey, University of Oxford; Assistant Editors: Professor Sir William Paton and G G Nahas, Columbia University and INSERM

Marihuana ' 84 contains reviews and papers on the latest marihuana research. The book deals with the pharmacology, metabolism and distribution of marihuana cannabinoids. It places emphasis on their adverse effects, particularly on reproductive functions and the immune response.

Papers cover the analysis of cannabinoids, focusing on the measurement of low levels of them and their metabolites in body fluids. Papers also review potentially therapeutic uses for cannabinoids, eg as anti-emetics in cancer chemotherapy.

Due May 1985; 800pp (approx):

0904147959 (hardback)

$£ 55.00 /$ US\$99.00 (provisional)

\section{OIRLPRESS}

Oxford - Washington DC

\section{David Park}

Time's Arrows: Scientific Attitudes

Toward Time.

By Richard Morris.

Simon \& Schuster: 1985. Pp.240. \$17.95.

IN THE philosophies of nature that most scientists seem to work out for themselves, time is something of a dark area. Time has extension analogous to space, but the impossibility of experiencing any instant more than once defies analogies - we face great difficulties if we try to define even so simple a word as "now" without tautology. An odd fact is that all this doesn't seem to matter. To use a concept such as $p \mathrm{H}$ or electric potential we need to understand more or less exactly what is meant, yet we readily navigate the shoals of time even if we cannot explain what we are doing. Our sense of time passing defies explanation in rational terms (how fast does it pass, then?); but we understand perfectly what we are doing when we use a clock.

This being the case, it has often been felt that there is some need for a further explanation of time, and yet in the absence of any practical difficulties one does not quite see what needs to be explained. In Time's Arrows Richard Morris adopts a reasonable programme: "We must discover what science can tell us about time before we can know what the relevant philosophical questions are". His remarks on the ancients are mercifully brief. The eye picks out the phrase "the philosopher Pythagoras" and again "Ancient peoples believed that time was cyclic in character" on an early page, and prepares to close in sleep, but pushing on we arrive at Galileo and the incorporation of a time variable, for the first time, in the description of physical phenomena (in this case free fall). Now we are safely in scientific territory. I do not want to encumber this review by enumerating the author's errors, but will mention three examples of the kind of thing that a reader ought not to have to bother with in reading an otherwise illuminating discussion.

- "Galileo was the first to realize that the acceleration of these [falling] bodies was a function of time." Since this remark is made in the context of motion with constant acceleration, $I$ can only attribute

- "Furthermore, Aristotle said, the velocity at which an object fell was proportional to its weight." This is indeed the conclusion that was widely drawn from passages in Aristotle (for example Physica $215^{\mathrm{a}}$ and $216^{\mathrm{a}}$ ), but these make no mention of falling bodies and "Aristotle said" is hardly the way to summarize them.

- In discussing free fall Morris attributes it to some demon in the pen. to Galileo the formula $d=1 / 2 a t^{2}$. Galileo never assigned a numerical value to acceleration and wrote no such formula. He did state that $d$ varies as $t^{2}$; this is an immediate consequence of the fourteenthcentury mean velocity rule. It would have been an interesting paragraph on the history of science if Morris had discussed the fact, surprising today, that the mediaeval scholars did not seek to apply their mathematical formulae to the natural world.

The next chapter is on "Calculus and the Idea of Determinism". After removing the sentence "Today, all of physics and most of higher mathematics are based on calculus", and placing it like a fishbone on the edge of the plate, the reader finds an excellent account of how the invention of the calculus made it possible to extrapolate present motions into the past and future so that the trajectory of a body, as a whole, could become a subject of study. The implications of Laplacian determinism are carefully discussed, though one would have hoped for at least a brief mention of the qualifications of the idea which the researches of the past decade on highly unstable motions have made necessary.

Morris next devotes two chapters to the enormous expansion of the old time-scale stemming from the work of Hutton and Lyell in geology, and Darwin and Wallace in biological evolution, and then moves on to modern physical science. The arrows of the book's title are five in number: the thermodynamic arrow of increasing entropy, the direction of cosmic expansion, the time-asymmetry of weak interactions, the electromagnetic arrow and the psychological arrow. The electromagnetic arrow, which requires that retarded solutions of the wave equation are the only ones to be retained, is the subject of an especially sensible discussion emphasizing that, in a universe containing $a b$ sorbing matter, either a diverging or a converging wave can be analysed in terms of either advanced or retarded functions; the predicted phenomena are the same in either case, and so the distinction lies outside physics in the mind of the analyst. The psychological arrow involves a real puzzle: how can one understand from all we know of time why consciousness singles out a single instant, now, for its attention?

The last chapters cover the fairly familiar territory of relativity and cosmology. As a whole, this is a somewhat uneven book, serious and thoughtful in places, jejune in others, disfigured by careless errors, but finally, I think, succeeding in what the author sets out to do: to suggest to the reader some of the thoughts that come to the mind of a scientist invited to focus on the word "time".

David Park is a Professor in the Department of Physics and Astronomy at Williams College, Williamstown, Massachusetts. 